\title{
Arthroskopische Refixation des TFCC bei ulnarer Ruptur
}

\author{
Arthroscopic Repair for Ulnar-sided Tears of the TFCC
}

\author{
Autoren \\ H.- G. Tünnerhoff ${ }^{1}$, M. Langer \\ Institute \\ ${ }^{1}$ Praxis für Hand - und Fußchirurgie, Belegabteilung Krankenhaus Marbach, Marbach \\ ${ }^{2}$ Unfallchirurgie, Universitätsklinik Münster, Münster
}

\section{Schlüsselwörter \\ - Arthroskopie \\ - TFCC Refixation \\ - DRUG \\ - Instabilität}

Key words

- arthroscopy

- TFCC repair

- DRUJ

- instability

eingereicht $\quad 6.7 .2014$

akzeptiert 17.7.2014

\section{Bibliografie}

DOI http://dx.doi.org/

10.1055/s-0034-1385937

Handchir Mikrochir Plast Chir 2014; 46: 286-294

(c) Georg Thieme Verlag KC

Stuttgart · New York

ISSN 0722-1819

Korrespondenzadresse

Dr. med. Heinrich-Geert

Tünnerhoff

Praxis für Hand - und

Fußchirurgie

Belegabteilung Krankenhaus

Marbach

Panoramastraße 9/1

71672 Marbach

HeinrichTuennerhoff@swol.de

\section{Zusammenfassung \\ $\nabla$}

Genaue anatomische und biomechanische Untersuchungen sowie klinische Erfahrungen haben in den letzten Jahren unsere Kenntnisse über die Funktion und Pathologie des distalen Radioulnargelenkes (DRUG) erweitert, sodass eine differenzierte Behandlung möglich ist. Bei Verletzungen reißt häufig der ulnare Teil des triangulären Faserkomplexes (TFCC). Die Entwicklung der verschiedenen arthroskopischen Techniken zur Rekonstruktion und der aktuelle Kenntnisstand werden im Folgenden dargestellt. Der ulnare Teil des TFCC ist in einen oberflächlichen, für die Stabilität weniger bedeutenden und einen wichtigeren, tiefen in der Fovea ansetzenden Teil zu unterscheiden. Beide Teile können zusammen oder jeweils einzeln abreißen. Schlüssel zur Diagnose sind die klinische Untersuchung und die Arthroskopie. Bei der klinischen Untersuchung ist eine Instabilität im Seitenvergleich und soweit möglich das Ausmaß der Instabilität festzustellen und auf extraartikuläre Begleitverletzungen zu achten. Arthroskopisch wird der TFCC von radiokarpal und mehr und mehr auch vom DRUG her dargestellt und palpatorisch beurteilt. Im Falle einer Avulsion in der Tiefe ist eine anatomisch korrekte Refixation an den Knochen angezeigt und nach den vorliegenden Studien und eigenen Erfahrungen erfolgreich. Im Falle einer sehr ausgeprägten Instabilität ist anzunehmen, dass das Ausmaß der Verletzung über den isolierten Abriss in der Fovea hinausgeht und eine alleinige Reinsertion nicht in allen Fällen ausreichend ist.

Die Arthroskopie des Handgelenkes hat in der Diagnostik und Behandlung der Erkrankungen des ulnaren Teiles des Handgelenkes und des distalen Radioulnargelenkes (DRUG) von Anfang an eine wichtige Rolle gespielt.

\section{Abstract \\ $\nabla$}

Accurately performed anatomic and biomechanical studies as well as clinical experience during the last years have widened our knowledge about function and pathology of the distal radioulnar joint (DRUJ) making a differentiated treatment possible. In cases of trauma a rupture of the ulnar part of the triangular fibrocartilaginous complex (TFCC) frequently occurs. An overview is given on the development of different arthroscopic techniques for repair and the state of the art is presented. The ulnar part of the TFCC is constituted of the superficial part which is less important for stability and the deep part which is anchored to the fovea of the ulnar head. This deep part is the most important stabiliser of the DRUJ. Each of these parts may rupture separately or both of them may rupture at the same time. Keystones for diagnosis are clinical examination and arthroscopy. On clinical examination instability of the DRUJ is to be evaluated by comparing it to the contralateral side. Furthermore, the degree of instability should be estimated. Extraarticular associated lesions must also be assessed clinically. On arthroscopy the TFCC may be evaluated from the radiocarpal joint as well as from its undersurface, from the DRUJ. In cases of avulsion from the fovea, an anatomic reconstruction with reinsertion of the deep part to the bone is indicated and may be performed successfully according to the published studies and our own experience. If a severe instability is found on clinical examination it is to be supposed that more stabilising structures - then only the radioulnar ligaments - are affected and reinsertion of the deep fibres to the fovea may not be sufficient.

Funktion und Stabilität des DRUG hängen angesichts geringer Formschlüssigkeit der Gelenkflächen in besonderem Maß von dynamischen und statischen, stabilisierenden Weichteilen ab. Zentrale Struktur zur Stabilisierung des DRUG und 
der Verbindung zur proximalen Handwurzel ist der trianguläre fibrokartilaginäre Faserkomplex (TFCC), in dem die ulnoradialen und ulnokarpalen Bänder verwoben sind [1] ( $\bullet$ Abb. 1).

Dieser kann traumatisch geschädigt werden, unterliegt oft aber auch erheblichen degenerativen Vorgängen. Verletzungen können komplex sein und Strukturen in der Umgebung des Gelenkes, besonders in Form von Rupturen der tiefen Schicht des sechsten Strecksehnenfaches (6. SSF), betreffen [2]. Operativ wurden debridierende, frühzeitig auch rekonstruktive Verfahren in Form von Nähten der peripheren ulnaren Risse und der radialen Abrisse durchgeführt. Inzwischen hat sich herausgestellt, dass bei der Behandlung der traumatischen Rupturen die klinisch zu diagnostizierende Instabilität des distalen Radioulnargelenkes (DRUG) eine wesentliche Rolle spielt und besonders die ulnarseitigen Abrisse näher zu unterteilen sind in solche, die zu einer wesentlichen Instabilität führen und solche, die nicht mit Instabilität verbunden sind. Ferner zeigte sich, dass die tiefe Insertion des TFCC isoliert abreißen kann.

Die Entwicklung der arthroskopischen Verfahren, der jetzige Stand und das praktische Vorgehen werden im Folgenden anhand der Literatur und eigener Ergebnisse dargestellt. Auf radialseitige Rupturen kann in dieser Arbeit nicht näher eingegangen werden. Ob arthroskopisch oder offen operiert wird, ist eher von untergeordneter Bedeutung, sofern die verletzten Strukturen korrekt rekonstruiert werden und beim Zugang wichtige Strukturen geschont werden.

\section{Entwicklung der arthroskopischen Verfahren \\ $\nabla$}

Die Einteilung der traumatischen Läsionen des TFCC nach Palmer [3] ist eine Domäne der Arthroskopie. Diese Klassifikation unterscheidet nach der anatomischen Lokalisation zentrale (IA), ulnare (IB), palmare (I C) und radiale (ID) Rupturen. Sie stützte sich anfangs auf arthrografische und intraoperativ erhobene Befunde. Die einfache Arthrografie des Handgelenkes hat sich inzwischen selbst bei Injektion aller 3 Kompartimente insgesamt als unsicher herausgestellt [4], ist jedoch speziell zum Nachweis einer Avulsion in der Fovea durchaus hilfreich [5]. Aufwendigere bildgebende Verfahren wie Arthro-CT und Arthro-MRT verbessern die Sensitivität und Spezifität erheblich [6]. Die Arthroskopie lässt kleinere Risse des TFCC feststellen und Knorpelschäden

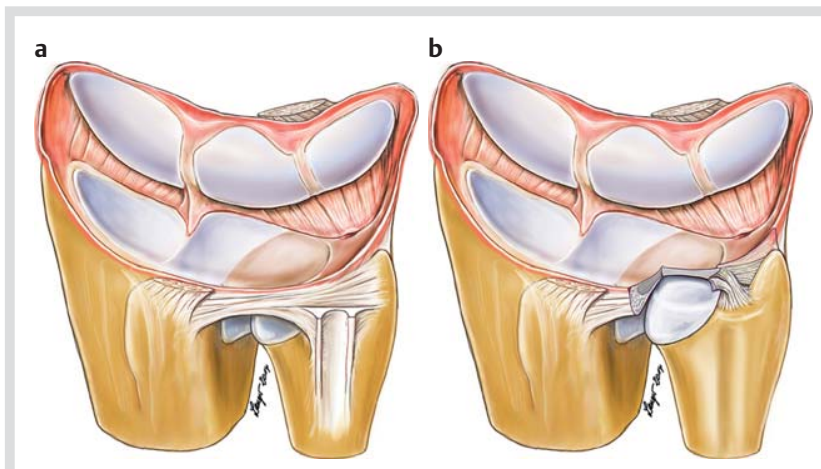

Abb. 1 Anatomie des TFCC: a Beachte die enge Verbindung zwischen oberflächlichem Teil des dorsalen ulnoradialen Bandes und dem Boden des 6. Strecksehnenfaches. b Die tieferen Schichten werden dargestellt. Beachte den überkreuzenden Verlauf der Fasern der tiefen Insertion in der Fovea. Dadurch werden ähnlich wie beim Kreuzband des Kniegelenkes abhängig von der Stellung des Gelenkes unterschiedliche Faserbündel zur Stabilisierung rekrutiert. sowie die Integrität und Spannung der intrinsischen und extrinsischen Bänder sowie des TFCC mit den ulnoradialen Bändern genau beurteilen und gilt als Goldstandard. Bestimmte Veränderungen wie ulnare Abrisse von der Kapsel lassen sich bei offenem Zugang zum Gelenk kaum verifizieren. Allerdings können auch arthroskopische Befunde diskret sein und dem flüchtigen Untersucher entgehen wie Spannungsverlust des TFCC durch Abriß in der Tiefe oder Frühstadien des ulnokarpalen Abutmentsyndroms.

Arthroskopisch operativ wurden zunächst Läsionen des TFCC debridiert und bei zentralen Rupturen Teilresektionen durchgeführt, bald aber auch Rupturen der Peripherie genäht [7]. Eine peripher gelegene Naht wurde als sinnvoll erkannt, da hier im Unterschied zum zentralen Bereich das Gewebe gut vaskularisiert ist [8]. Die arthroskopische Darstellung des TFCC erfolgt in der Regel vom Radiokarpalgelenk her. Aus dieser Sicht neigt man dazu, den TFCC als 2-dimensionale Struktur zu betrachten.

Mit den ersten Nahttechniken wurde versucht, den Abriss des TFCC durch Refixation an die Kapsel und damit den Boden des 6. Strecksehnenfaches zu rekonstruieren und die Spannung des TFCC in transversaler Ebene wiederherzustellen [9]. Als etabliertes offenes Verfahren stand dem die offene transossäre Reinsertion des ulnarseitigen TFCC in die Fovea gegenüber [10]. Experimentelle Untersuchungen zeigten, dass die Stabilität, die bei Naht an den Boden des 6. SSF und bei transossärer Reinsertion erzielt werden kann, sich nicht unterscheidet [11]. In einem klinischen Vergleich von offenen und arthroskopischen Refixationen bei periphereren Rupturen wurden eine offene transossäre Fixationstechnik des TFCC in die Fovea einer arthroskopischen Refixation an die Kapsel gegenübergestellt, ohne dass signifikante Unterschiede festgestellt werden konnten. Allerdings wurden nach beiden Verfahren in insgesamt 17\% der Fälle Re-Operationen wegen Instabilität erforderlich, durchgeführt als Bandplastik mit freiem Sehnentransplantat [12]. In mehreren Publikationen wurde über Resultate nach arthroskopischer Refixation an den Boden des 6. SSF in verschiedenen technischen Variationen berichtet, teilweise mit insgesamt zufriedenstellenden Resultaten $[9,13,14]$, teilweise mit guten und sehr guten Resultaten in $66[15,16]$ bis $75 \%$ der Fälle [17]. Nicht berücksichtigt wurde bei diesen Studien, dass oberflächlicher und tiefer Teil des TFCC von unterschiedlicher Struktur sind und unterschiedliche Funktionen besonders bezüglich der Stabilität des DRUG haben. Palmer [3] hat in seiner bahnbrechenden Arbeit die ulnaren Rupturen als Ausrissverletzungen des TFCC aus der Fovea beschrieben und einen Zusammenhang mit einer Instabilität des DRUG angenommen. Eigene Nachuntersuchungen nach Refixation des oberflächlichen Teiles des TFCC zeigten nur in $2 / 3$ der Fälle gute und sehr gute Ergebnisse. Das Resultat der OP hing wesentlich damit zusammen, ob das DRUG als stabil oder instabil eingeschätzt wurde. Eine präoperativ festgestellte Instabilität konnte durch die Naht nicht zuverlässig gebessert werden [18]. Ein ähnlicher Zusammenhang wurde auch bei der Nachuntersuchung der von Wedemeier et al. mit Refixation des TFCC an den Boden des 6. SSF behandelten Patienten festgestellt [19].

\section{Aktueller Stand}

In der Zwischenzeit wurden durch experimentelle, biomechanische Studien und anatomische und histologische Untersuchungen die Biomechanik des DRUG und die Funktion der einzelnen Anteile des TFCC genauer beschrieben. $\mathrm{Zu}$ unterscheiden ist in oberflächliche und tiefe, dorsale und palmare ulnoradiale Bänder. Aufgrund der „Inkongruenz“ der Gelenkflächen des DRUG 
mit unterschiedlichen Krümmungsradien muss dieses besonders in Pronation und Supination durch Weichteilstrukturen stabilisiert werden. Die Bandanteile des TFCC gelten als primäre Stabilisatoren, wobei als wichtigste das palmare tiefe ulnoradiale Band in Pronation, das dorsale tiefe ulnoradiale Band in Supination angespannt werden und in Funktion treten [20-23]. Hagert hat die funktionelle Bedeutung der tiefen Insertion des TFCC - fixed point concept - als Dreh- und Angelpunkt der Unterarmrotation unter gleichzeitiger Stabilisierung der proximalen Handwurzelreihe hervorgehoben [24]. Nakamura hat die einzelnen Anteile des TFCC histologisch aufgearbeitet und gezeigt, dass nur an der tiefen Insertion des TFCC am Knochen sowie dorsal und palmar am Radius sich Sharpey'sche Fasern nachweisen lassen und vorwiegend den hier ansetzenden Fasern also die Funktion eines stabilisierenden Bandes zuzuschreiben ist [25]. Wichtig ist, dass der TFCC als 3-dimensionale Struktur erkannt wird und die tiefen Fasern zunächst senkrecht aufsteigen.

Der Ursprung der tiefen ulnoradialen Fasern in der Fovea liegt näher am Radius, in der Rotationsachse des DRUG, der Ursprung der oberflächlichen Fasern weiter weg vom Radius, sodass der Ansatzwinkel der tiefen Fasern größer und damit effektiver ist als der der oberflächlichen Fasern, die in einem spitzeren Winkel am Radius ansetzen [26]. Klinische Beobachtungen haben ferner gezeigt, dass der tiefe Teil des TFCC isoliert abreißen kann und ulnare Bandrupturen in unterschiedlichem Ausmaß den tiefen und oberflächlichen Bandanteil betreffen können ( $\bullet$ Abb. 2). Atzei hat eine Klassifikation angegeben, nach der die ulnaren traumatischen Läsionen des TFCC in rein oberflächliche Abrisse von der Kapsel ohne wesentliche Instabilität des Gelenks, alleinige Abrisse der tiefen Insertion vom Knochen mit mäßig ausgeprägter, aber deutlich erkennbarer Instabilität des Gelenkes und kombinierte Verletzungen zu unterteilen sind. Als zusätzliche Gruppe unterscheidet Atzei Patienten mit fortgeschrittener Destruktion der in der Tiefe ansetzenden ligamentären Anteile und solche mit etablierter Arthrose des DRUG. Bei diesen Patienten ist eine einfache Reinsertion des TFCC nicht ausreichend, sondern es muss eine Bandplastik bzw. eine Rettungsoperation für das DRUG durchgeführt werden [27]. Außerdem sind isolierte Längsrisse des ulnotriquetralen (UT-) Bandes zu beobachten, die ulnarseitige Schmerzen ohne Instabilität verursachen können [28].

Moritomo [29] hat aufgrund von kinematischen in-vivo-Untersuchungen eine Hypothese zum Verletzungsmechanismus aufgestellt, nach der ein Hyperextensionstrauma des Handgelenkes durch forcierte Radial- und Extensionsbewegung des Karpus zusammen mit forcierter Pro- oder Supination zu einer ver-
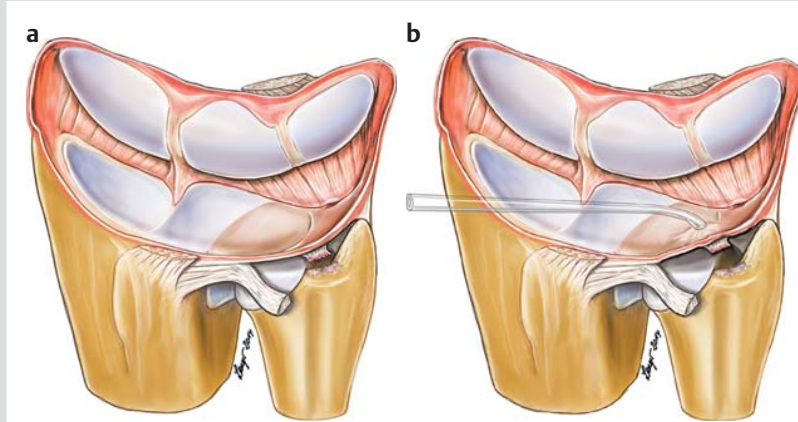

Abb. 2 Avulsion der tiefen Fasern in der Fovea. Der TFCC hat an Spannung verloren a und lässt sich mit dem Sauger von radiokarpal deutlich sichtbar anheben $\mathbf{b}$. Der dorsale radioulnare Bandapparat ist aus rein zeichnerischen Gründen herausgeschwenkt. mehrten Spannung der in der tiefe ansetzenden Fasern des TFCC und schließlich zum Abriss führen kann. Der palmare oberflächliche Teil kann dabei ebenfalls abreißen, wogegen der oberflächliche dorsale Teil intakt bleibt. Das typische Rotationstrauma (hängenbleibende Bohrmaschine) führt dagegen zusätzlich zu Verletzungen des oberflächlichen, dorsalen, ulnoradialen Bandes und unter Umständen zu Läsionen der direkt darüber verlaufenden ECU-Sehnenscheide [30]. Zu ergänzen ist, dass auch radialseitige Abrisse zu symptomatischer Instabilität führen können, sofern der ligamentäre Anteil betroffen ist [31].

Verschiedene arthroskopische Verfahren zur radialen Reinsertion wurden angegeben [32].

\section{Praktisches Vorgehen bei traumatischer Ruptur des TFCC $\nabla$}

Anamnese

In der Regel ist ein Trauma in der Vorgeschichte zu eruieren. Meistens wird ein Sturz auf das überstreckte Handgelenk entweder nach vorne in Pronation oder nach hinten in Supination berichtet, seltener das früher als charakteristisch angesehene Rotationstrauma (Hängenbleiben mit der Bohrmaschine) [30]. Nur gelegentlich können sich Patienten nicht mehr an einen Unfall erinnern. Gezielt zu fragen ist nach früheren Verletzungen, insbesondere Unterarmfrakturen, die unter einer eventuell nur geringen Fehlstellung ausgeheilt sein können.

\section{Klinischer Befund}

Erster Hinweis ist der vom Patienten ulnar lokalisierte Schmerz. Abhängig von der Art der Ruptur ist eine Instabilität im Verschiebetest zwischen Ulna und Radius, dem Ballottement-Test, festzustellen. Dabei wird die Ulna mit einer Hand fixiert und der Radius mit der Handwurzel aus der Mittelstellung heraus nach dorsal und palmar geschoben. Zunächst wird in Neutralstellung, dann in Pronation und Supination untersucht. Es muss mehrfach untersucht werden, da das Ergebnis leicht durch Muskelanspannung des Patienten verfälscht werden kann - auf jeden Fall auch unmittelbar präoperativ nach Anlage der Anästhesie im Seitenvergleich. Die sorgfältige Untersuchung in Neutralrotation, Supination und Pronation gibt einen gewissen Anhalt dafür, welche Strukturen verletzt sind. Besteht eine Instabilität aus der Neutralstellung nur in eine Richtung kann eine isolierte Läsion des palmaren oder seltener dorsalen ulnoradialen Bandes vorliegen [26]. Das Handgelenk wird bei der Prüfung der Stabilität zunächst in Neutralstellung gehalten. In Radialduktion kommt es zu einer Stabilisierung des DRUG, auch bei Abriss der tiefen Insertion in der Fovea, sofern die ECU Sehnenscheide intakt ist. In Extensionsstellung des Handgelenkes werden die ulnokarpalen Bänder angespannt und es kommt zu einer effektiven, klinisch deutlichen Stabilisierung des DRUG, die aber nicht eintritt wenn die tiefen Fasern des TFCC in der Fovea abgerissen sind [33]. Fehlstellungen des Knochens z.B. nach einer metaphysären Radiusfraktur in der Adoleszenz sind ebenfalls mit einer unidirektionalen Instabilität verbunden. Schon überhaupt festzustellen, ob eine Instabilität vorliegt, ist schwierig und bedarf einer wiederholten Untersuchung. Darüber hinaus ist es erforderlich das Ausmaß der Instabilität abzuschätzen, da bei isolierter Verletzung der tiefen Insertion des TFCC eine mäßige, bei darüber hinausgehender Verletzung eine ausgeprägte Instabilität zu erwarten ist $[5,20]$. 
Bei Abriss nur des oberflächlichen Teiles des TFCC ist keine Instabilität zu erwarten. Bei Abriss des TFCC in der Fovea ist häufig ein umschriebener Druckschmerz unmittelbar über der Abrissstelle, also zwischen der ECU- und der FCU-Sehne, etwas palmar des Processus styloideus der Ulna (PSU) festzustellen. Dieses Fovea-Zeichen ist nach Tay [34] sehr spezifisch entweder für einen Abriss des TFCC in der Fovea oder, sofern keine Instabilität besteht, für eine Längsruptur zwischen äußerstem ulnarem und radialem Anteil des UT-Bandes.

Der ulnokarpale Stress-Test bzw. das Ulna-Grinding-Manöver provozieren bei der rein traumatischen ulnarseitigen Ruptur eher geringe bis mäßige Beschwerden im Unterschied zum ulnaren Impaktion-Syndrom. $\mathrm{Zu}$ achten ist auf mögliche Begleitverletzungen, besonders des LT-Bandes (Ballottement-Test zwischen Lunatum und Triquetrum nach Reagan [35] oder ShearTest des Pisiforme und Triquetrum gegenüber dem Lunatum nach Kleinman). Ein Druckschmerz ist in diesen Fällen etwas weiter distal als beim Fovea-Zeichen, nämlich über dem Triquetrum, auszulösen.

Auch Begleitverletzungen der ECU-Sehnenscheide oder selten eine stenosierende Tendovaginitis sind $\mathrm{zu}$ berücksichtigen $[36,37]$. Die ECU-Sehne kann genau palpiert und mit den entsprechenden Belastungsmanövern (Ulnarduktion gegen Widerstand, Dehnung in Supination und Radialduktion und Synergietest [38], der sehr hilfreich ist) geprüft werden. Bei schlanken Menschen kann man tasten, ob die Sehne im Seitenvergleich vermehrt beweglich ist und eine Subluxation provoziert werden kann, was am besten in maximaler Supination und Ulnarduktion gegen Widerstand geschieht [37].

Der Schmerz wird nach meiner Erfahrung allerdings vom Patienten nicht immer ulnar lokalisiert, sondern öfters auch als diffus oder radialseitig empfunden. Manche Patienten werden zunächst wegen radialer Handgelenksbeschwerden oder einer Sehnenscheidenentzündung behandelt, bis sich herausstellt, dass die Ursache der Beschwerden die durch den Abriss der tiefen Fasern des TFCC in der Fovea ausgelöste Instabilität der proximalen Handwurzelreihe ist.

\section{Bildgebende Verfahren}

Erforderlich ist ein exakt eingestelltes Röntgenbild des Handgelenkes in 2 Ebenen zur Beurteilung der ulnaren Varianz. Eine Belastungsaufnahme kann helfen, ein dynamisches Ulna-Impaktion-Syndrom abzuklären. Besonders wichtig ist darauf zu achten, dass die knöcherne Kongruenz des DRUG intakt ist. Auch geringe Fehlstellungen nach einer distalen Radiusfraktur können Instabilitäten und ulnare Handgelenksbeschwerden verursachen $[39,40]$. Leichte Fehlstellungen nach einer weiter proximal gelegenen Radiusfraktur können ebenfalls zu einer symptomatischen Instabilität des DRUG führen. Auch verbliebene Radialtranslokationen des distalen Fragmentes bei sonst korrekter Stellung der Fraktur können eine Instabilität des DRUG verursachen [41]. Bei entsprechender Anamnese muss der Unterarm ebenfalls radiologisch dargestellt werden.

Die Bedeutung weiterer bildgebender Verfahren ist nicht endgültig geklärt. Die Genauigkeit des konventionellen MRT ist bezüglich der Verletzungen des TFCC, besonders unter den Bedingungen der täglichen Praxis, noch unzureichend [42]. In differenzialdiagnostischer Hinsicht und zur Abklärung von Begleitverletzungen kann dieses jedoch hilfreich sein. Die Informationen durch hochauflösendes MRT, Arthro-CT und Arthro-MRT sind auch für den versierten Arthroskopeur wertvoll, da sie in der Praxis helfen können, nicht ganz eindeutige arthroskopische Befunde zu klären.
Insbesondere partielle Rupturen der tiefen Insertion des TFCC sind im MRT erkennbar, arthroskopisch aber nur schwierig darzustellen. $\mathrm{Zu}$ wünschen ist eine enge Zusammenarbeit zwischen Radiologe und Handchirurg [43]. Läsionen der ECU-Sehnenscheide können im MRT in den transversalen Schichten oder sonografisch erkannt werden [44].

\section{Konservative Behandlung}

Sowohl in der akuten Phase wie nach länger zurückliegendem Trauma ist zunächst eine konservative Behandlung angezeigt. Unmittelbar nach einer Verletzung des Handgelenkes durch Extensions- oder Rotationstrauma wird das Gelenk durch eine Schiene immobilisiert. Wie lange und welche Gelenke immobilisiert werden sollten, ist nicht durch Daten zu belegen. Praktikabel erscheinen 4-6 Wochen in einer ulnar umgreifenden Unterarm-Schiene. Nach Park et al. ist bei ca. der Hälfte der Patienten mit klinisch diagnostizierter Verletzung des TFCC eine konservative Behandlung ausreichend [45]. Patienten mit deutlicher Instabilität des DRUG wurden in dieser Studie nicht berücksichtigt. Zur Stabilisierung des DRUG in der postakuten Phase kommt eine dorsopalmare Manschette in Betracht, die den Unterarm in der transversalen Ebene komprimiert und Ellbogen sowie Handgelenk freilässt, ähnlich der von Sarmiento und Mitarb. [46] angegebenen Schiene. Der Effekt einer solchen Schiene wurde experimentell nachgewiesen [47]. In der chronischen Situation, wenn das Trauma Wochen oder Monate zurückliegt, sind propriozeptive Übungen zur muskulären Stabilisierung des DRUG angezeigt.

\section{Operative Behandlung}

Wenn nach Ausschöpfung konservativer Maßnahmen ulnarseitige Handgelenksbeschwerden persistieren, ist über bildgebende Verfahren hinaus eine arthroskopische Abklärung und Behandlung zweckmäßig. Ob eine operative Intervention erforderlich ist, hängt sehr wesentlich von der Situation und Aktivität des Patienten ab. Eine spontane Besserung des Beschwerdebildes ist auch nach mehreren Monaten möglich. Bei klinisch deutlicher Instabilität ist ein operativ arthroskopisches Vorgehen mit dem Ziel, in der gleichen Sitzung eine Refixation des TFCC durchzuführen, eher frühzeitiger angezeigt als bei einem stabilen DRUG. Moritomo berichtet allerdings, dass von 35 Patienten mit nachgewiesener Avulsion des TFCC in der Fovea 16 allein unter konservativen Maßnahmen beschwerdefrei wurden. Die Behandlungsergebnisse hängen nach eigener Erfahrung nicht unbedingt vom Zeitpunkt der Operation ab. Nakamura et al. beobachtete dagegen schlechtere Ergebnisse, wenn die arthroskopische Refixation später als 7 Monate, eine offene Operation später als $1 \mathrm{Jahr}$ nach dem Trauma erfolgte. Eine Rolle könnte spielen, ob der Knochen ausreichend angefrischt und das Narbengewebe debridiert wird. Jedenfalls bleiben die ulnoradialen Bänder durch ihre Verwebung mit dem TFCC nach einer Avulsion aus der Fovea für längere Zeit in situ und sind auch nach mehreren Monaten noch für eine Reinsertion geeignet.

\section{Arthroskopischer Befund}

$\nabla$

\section{Radiokarpalgelenk}

Arthroskopisch wird der ulnare Teil des Handgelenkes in üblicher Technik unter Distraktion mit einem 2,3 oder 1,9 mm Arthroskop über das 3/4-Portal dargestellt. Der TFCC wird mit dem Tasthäkchen über das 6R- oder 4/5-Portal untersucht und der 
Knorpel besonders am ulnaren Pol des Lunatums beurteilt und mit dem Häkchen palpiert. Das LT-Band lässt sich am besten über das 6R-Portal einsehen, zur Palpation oder zum Debridement kann das 6U-Portal verwendet werden.

Komplette ulnare Abrisse und Rupturen des oberflächlichen Teiles sind arthroskopisch leicht festzustellen. Isolierte tiefe Läsionen sind bei der Arthroskopie von radiokarpal nur indirekt festzustellen, da die sichtbaren oberflächlichen Anteile des TFCC erhalten sind. Man sieht dann nur, dass die Spannung des TFCC herabgesetzt ist (Trampolin-Test) und sich dieser vermehrt von der Unterlage abheben lässt (Haken-Test). Eine auffällige Synovialitis kann den Verdacht auf eine tiefergelegene Läsion wecken. Auch beim Arbeiten mit dem Shaver wird sichtbar, dass durch Sog der TFCC von der Unterlage abgehoben wird (Saug-Test) ( $\bullet$ Abb. 2b, 3). Elementar wichtig ist, dass klinisch, auch intraoperativ, bei Abriss der tiefen Insertion eine Instabilität des DRUG im Seitenvergleich erkennbar ist.
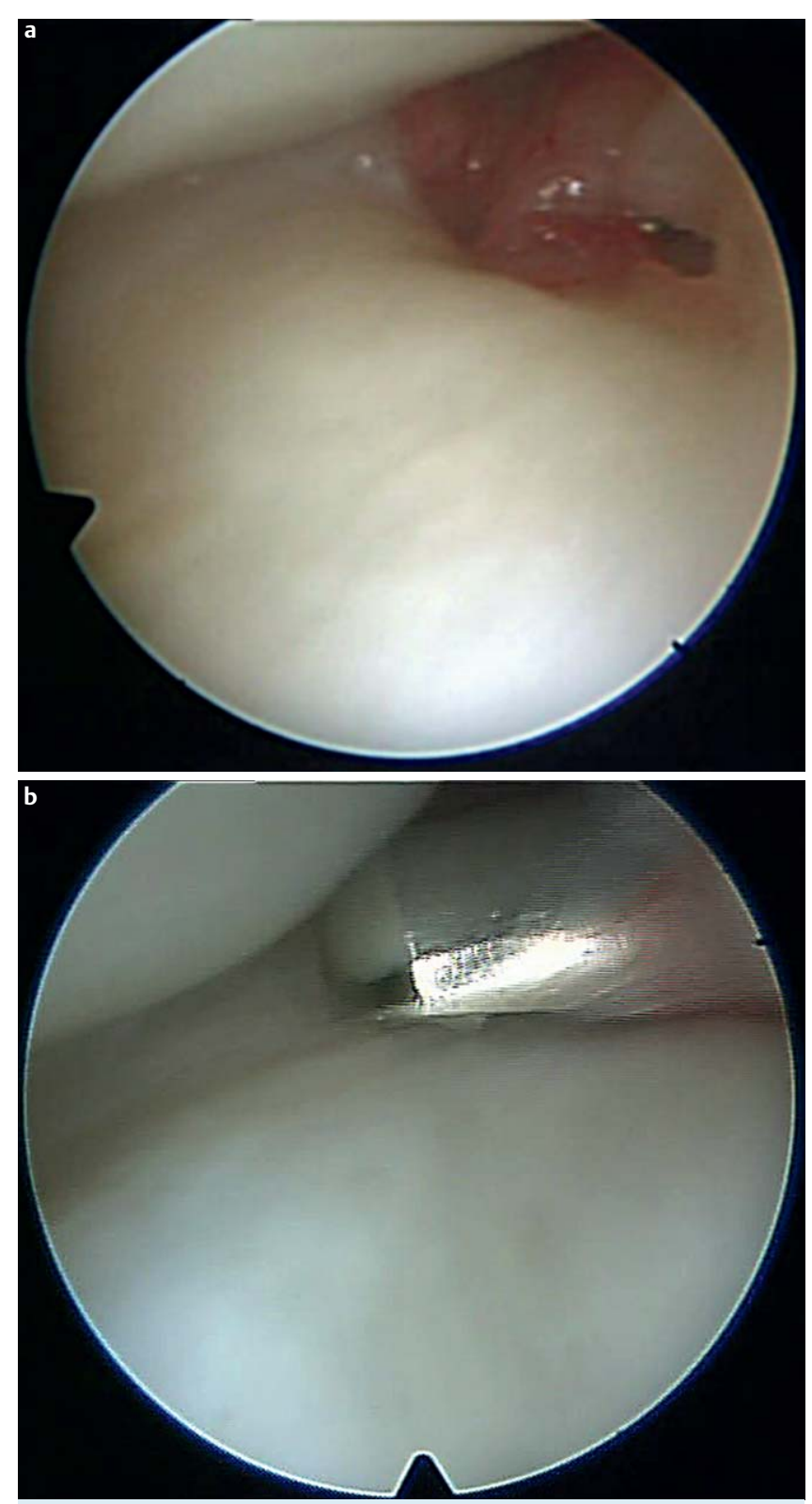

Abb. 3 Rechtes Handgelenk, Arthroskopie von radiokarpal: Abriss der tiefen Insertion des TFCC. Dieser ist vermehrt verschiebbar a und mit dem Sauger abzuheben $\mathbf{b}$. Beachte die ulnarseitige Synovialitis.
Radiale Rupturen können in rein zentrale Einrisse, die ähnlich wie IA-Rupturen debridiert werden, und Abrisse, die den ligamentären Anteil betreffen, unterschieden werden. Die ulnoradialen Bänder können dorsal, palmar oder durchgehend am Radius abreißen. In diesen Fällen ist ein reines Debridement nicht ausreichend [31].

\section{Distales Radioulnargelenk}

Über die Darstellung des TFCC von radiokarpal hinaus lässt sich auch dessen Unterseite durch eine Arthroskopie des DRUG darstellen. Aus dieser Perspektive sieht man die Insertion der ulnoradialen Bänder in der Fovea direkt. Man verschiebt die Weichteile über dem 6R-Portal etwas nach proximal, fährt in Neutralstellung bis mäßiger Supination unter leichtem Nachlassen der Distraktion mit einer kleinen Klemme unter den TFCC und richtet so ein distales dorsales DRUG-Portal ein [49]. Dieses Manöver gelingt in der Regel ohne Probleme, wenn die tiefe Insertion des TFCC abgerissen ist, bei intaktem TFCC kaum. Es gibt daher einen Hinweis auf eine Ruptur der tiefen Fasern. Es kann darüber hinaus beurteilt werden, ob ein kompletter oder partieller Abriss der Fasern in der Tiefe vorliegt. Gelegentlich sieht man, dass die ulnoradialen Bänder in der Tiefe durch insuffizientes Narbengewebe oder eine leere synoviale Hülle ersetzt sind [48]. Nach der Struktur des abgerissenen Bandes kann man versuchen abzuschätzen, wieweit dieses für eine Reinsertion geeignet ist, falls die Verletzung schon mehrere Monate zurückliegt ( $\bullet$ Abb. 4).

\section{Arthroskopische Therapie \\ $\nabla$}

Therapeutisch ist in erster Linie die Stabilität durch Refixation der abgerissenen Bänder, bei ulnarseitigen Rupturen also der tiefen Fasern in der Fovea wiederherzustellen. Diese Reinsertion kann arthroskopisch als transossäre Naht oder mittels eines Nahtankers oder auch offen durchgeführt werden.

Der Nahtanker wird über eine kurze etwas palmar der Fovea gelegene Inzision, auch als „direct foveal portal“ bezeichnet, direkt in die Fovea eingebracht.

Zweckmäßig ist ein Schraubanker, der mit 2 Fäden armiert ist, sodass gezielt der dorsale und der palmare Teil des ulnaren TFCC gefasst werden kann. Atzei berichtet über 37 Patienten, die nach dieser Technik versorgt wurden [27]. 18 Patienten wurden nachuntersucht, 14 zeigten komplette Abrisse, 4 einen isolierten Abriss in der Tiefe. Der Schmerz ging von 8,2 auf 1,2 auf der VAS zurück, der DASH-Score lag bei durchschnittlich 10,5. Der Mayo Modified Wrist Score (MMWS) war exzellent bei 14, gut bei 3 und mäßig bei einem Patienten.

Der Faden kann auch transossär durch mittels K-Drähten angelegte Knochenkanäle schräg von proximal ulnar in die Fovea geleitet und mithilfe von Kanülen und Schlaufen durch den ulnaren Rand des TFCC geführt werden. Nakamura und Mitarb. berichteten über 24 Patienten, bei denen der TFCC arthroskopisch in dieser Art refixiert wurde [48]. Nach einem eigenen DRUG-Score wurden 13 exzellente, 3 gute, 4 mäßige und 4 schlechte Ergebnisse erreicht. Das Ergebnis war abhängig vom Zeitraum zwischen Unfall und Operation. Gute und sehr gute Ergebnisse wurden bei Patienten erreicht, die innerhalb von 7 Monaten nach dem Unfall arthroskopisch operiert wurden, mäßige und schlechte bei späterer Operation. Bei offener Refixation mit Zugang über das 6. SSF und Anlage von 2, an der 3-dimensionalen Anatomie und Verlaufsrichtung der Fasern orientierten Matratzennähten konnten auch nach längeren Zeiträumen gute Ergebnisse erzielt werden: 

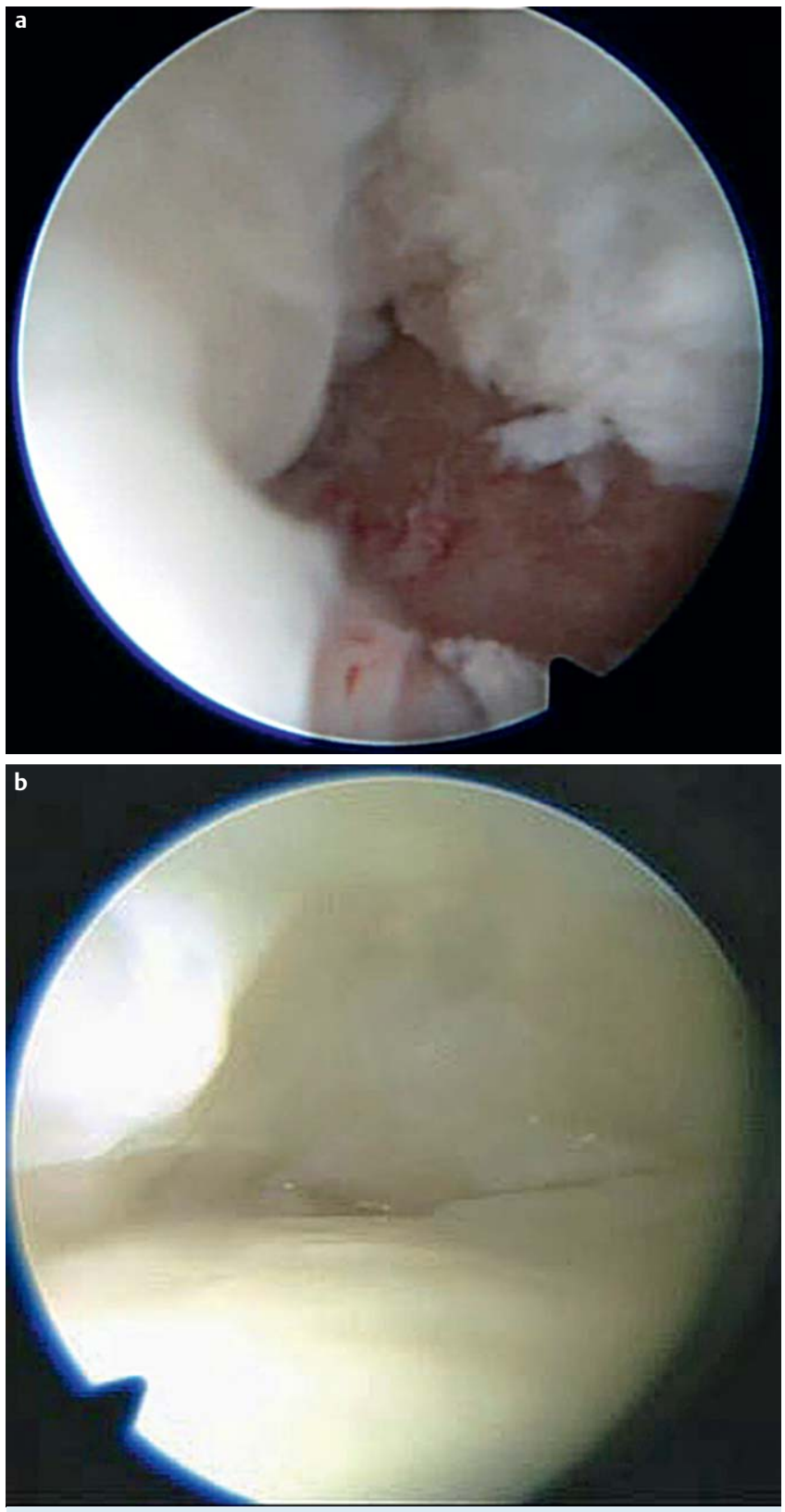

Abb. 4 a Arthroskopie des DRUG, linkes Handgelenk: frischer Ausriss des TFCC aus der Fovea. b Arthroskopie des DRUG, rechtes Handgelenk: älterer Ausriss der Fasern aus der Fovea. Diese ist von Narbengewebe bedeckt, die in der Tiefe ansetzenden Fasern fehlen. Unten Caput ulnae, oben Unterseite des TFCC.

von 66 Patienten zeigten 56 ein exzellentes, 6 ein gutes, 2 ein mäßiges und 2 ein schlechtes Resultat. Die Stabilität des DRUG wurde bei 17 Patienten der arthroskopischen Gruppe und 56 Patienten der offen operierten Gruppe wiederhergestellt. Shinohara et al. berichten nach Operation in der gleichen arthroskopischen Technik über 11 Patienten mit einem MMWS von durchschnittlich 94 Punkten [50]. Es wurden 6 exzellente, 4 gute und ein mäßiges Resultat erreicht. 7 Patienten waren schmerzfrei, 4 hatten leichte Schmerzen unter Belastung. Zu erwähnen ist eine Reinsertionstechnik mit gleichzeitiger Erzeugung einer blutenden Knochenwunde durch Anlegen eines 2,9 mm Bohrkanals in die Fovea, wobei der Kanal wie die K-Drähte von proximal ulnar schräg in der Fovea mündend angebracht wird. Mit dieser Technik ist das Ein- bringen der Fäden durch den großen Bohrkanal einfach durchzuführen. Bei 12 Patienten wurden mit dieser Technik ein durchschnittlicher MMWS von 92,5 und DASH-Score von 7,7 Punkten erreicht [51]. Bei der offenen Technik kann man von dorsal oder palmar zugehen. Von dorsal kann die Reinsertion mit einer Doppelung der dorsalen Kapsel verbunden werden, was bei ausgeprägten Instabilitäten hilfreich sein kann. Bei palmarem Zugang können die in der dorsalen Kapsel liegenden propriozeptiven Elemente geschont werden [30,52].

Isolierte oberflächliche Einrisse des TFCC ohne Instabilität können an den Boden des 6 . SSF refixiert werden. Wysocki et al. berichten über 29 Patienten, von denen 25 nachuntersucht wurden [53]. Der Schmerz ging von durchschnittlich 5,4 auf 0,9 auf der VAS zurück, der DASH-Score besserte sich von 38 auf 9. 21 Patienten sahen die Operation als erfolgreich an. Wolf et al. haben bei 49 Patienten wegen einer Palmer-IB-Läsion ohne Instabilität den TFCC arthroskopisch refixiert [54]. Es wurden 46 Patienten nach durchschnittlich 11 Monaten und 4,8 Jahren nachuntersucht. Der DASH-Score besserte sich von präoperativ 35 auf kurz postoperativ 22 und schließlich 14 Punkte.

Zur Behandlung der oberflächlichen Abrisse kommt auch das alleinige Debridement in Betracht, wobei einerseits über gute Ergebnisse berichtet wird [55], andererseits festgestellt wird, dass das alleinige Debridement nicht effektiv ist [56]. Möglicherweise hängen diese unterschiedlichen Ergebnisse mit der Indikationsstellung und einer unterschiedlichen Ausdehnung der Rupturen zusammen.

\section{Eigene Technik und Ergebnisse \\ $\nabla$}

Die eigenen Erfahrungen mit der anfangs allgemein praktizierten Technik, bei ulnarem Riss den TFCC an den Boden des 6. SSF zu refixieren, waren unbefriedigend. Das Ergebnis hing offensichtlich mit einer persistierenden Instabilität zusammen. Ich habe daraufhin die Technik geändert, um die tiefen Fasern anatomiegerecht durch 2 transossäre Nähte in die Fovea zu reinserieren.

Im Distraktionsturm werden nach kurzer Längsinzision streng ulnar über dem PSU die subkutanen Weichteile vom Retinakulum unter Schonung des R. dorsalis des N. ulnaris abgeschoben. Das Retinakulum wird inzidiert und auf dem Kochen um die Basis des PSU dorsal und palmar präpariert, sodass die Fovea von dorsal und palmar ertastet werden kann. Dabei wird kurzstreckig das 6. SSF eröffnet. In Supination wird der Knochen in der Fovea von palmar mit einem feinen Luer bis auf die Spongiosa angefrischt. Mit dem Zahnarzthäkchen wird von dorsal und palmar her überprüft, dass der harte kortikale Knochen reseziert ist und eine Rinne in der Fovea gebildet wurde. Ein Knochenkanal wird mit einem 1,2 mm K-Draht von proximal ulnar nach distal radial gebohrt. Der Draht zielt dabei zentrisch auf die angefrischte Fovea ( $\bullet$ Abb. 5a) und wird dann unter arthroskopischer Kontrolle durch den ulnaren Teil des TFCC bis gerade eben in das Gelenk gebohrt. Die Spitze des Drahtes soll im Gelenk am Übergang von dorsalem und palmarem Bandapparat nahe dem Rand des TFCC erscheinen. Der K-Draht wird entfernt und eine Kanüle der Größe 1 (20G) mit eingezogener Fadenschlinge in den Bohrkanal eingebracht, deren Spitze analog zum K-Draht am Übergang von dorsalem und palmarem Bandapparat den TFCC nahe an seinem ulnaren Rand perforiert. Palmar des PSU wird unter Knochenkontakt eine Kanüle Größe 2 (21 G) mit 
einem einliegenden 2/0 PDS-Faden von proximal durch den TFCC gestochen, sodass die Kanülenspitze am palmaren Rand des palmaren radioulnaren Bandes sichtbar wird. Der Faden wird mit der Fadenschlinge gefasst und transossär durch die Ulna herausgezogen ( $\bullet \mathbf{A b b} . \mathbf{5 b}$ ), durch Zug auf korrekte Lage geprüft und angeklemmt ( $\boldsymbol{O} \mathbf{A b b} . \mathbf{5 c})$. Nun wird wiederum in EinwärtsAuswärts-Technik eine zweite Naht zur Reinsertion der dorsalen TFCC-Anteile eingezogen. Hierzu wird eine mit einem 2/0 PDSFaden armierte Kanüle Größe 2 (21 G) durch das zentrale Bohrloch in den TFCC eingestochen und der Faden dann mittels einer in eine Kanüle Größe 1 (20G) eingezogenen Fadenschlinge gefasst und dorsal um den PSU herausgeleitet. Beide Nähte werden ohne Distraktionszug, in Neutralrotation des Unterarmes, unter maximaler Spannung auf dem Knochen geknüpft ( $\bullet$ Abb. $\mathbf{5 d}$ ),

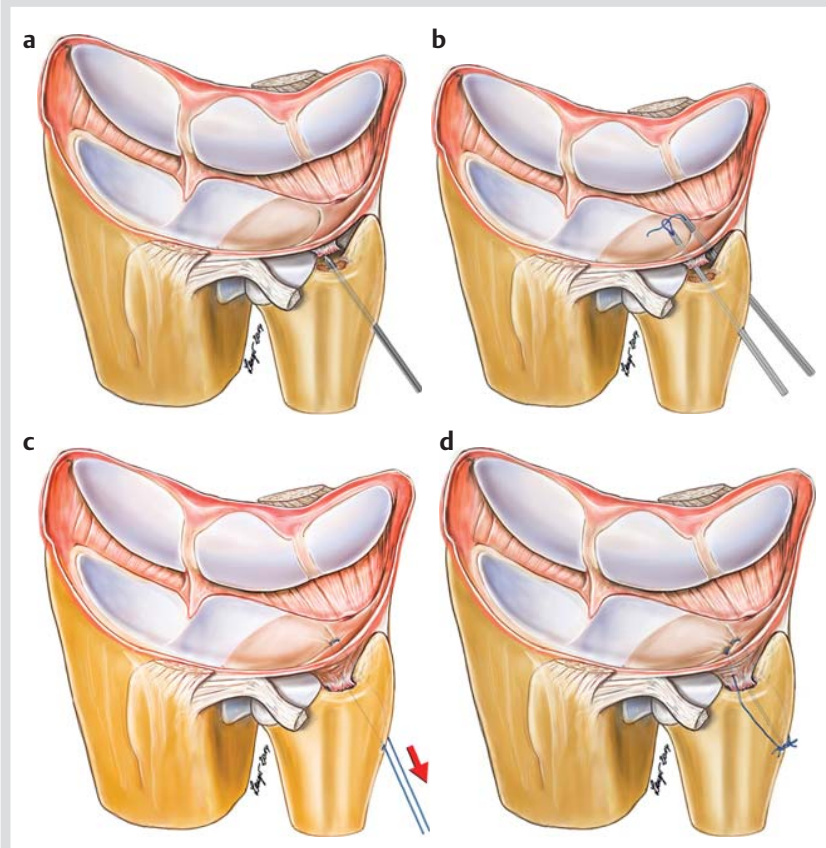

Abb. 5 Technik der transossären Insertion: a Der Knochen in der Fovea wird mit einem kleinen Luer bis auf die Spongiosa angefrischt, sodass eine Rinne entsteht, in die der TFCC hineingezogen werden kann. Bohren eines Knochenkanals von proximal ulnar nach distal radial mit einem 1,2 mm KDraht, dessen Spitze zentral in der Knochenrinne zu liegen kommt (a) und dann unter arthroskopischer Kontrolle durch den ulnaren Teil des TFCC bis gerade eben in das Gelenk gebohrt wird. Die Spitze des Drahtes soll im Gelenk am Übergang von dorsalem und palmarem Bandapparat nahe dem Rand des TFCC erscheinen. b Der K-Draht wird entfernt und eine Kanüle der Größe 1 (20 G) - gelb- mit eingezogener Fadenschlinge in den Bohrkanal eingebracht, deren Spitze analog zum K-Draht am Übergang von dorsalem und palmarem Bandapparat den TFCC nahe an seinem ulnaren Rand perforiert. Palmar des PSU wird unter Knochenkontakt eine Kanüle der Größe 2 (21 G) - grün - mit einem einliegenden 2/OPDSFaden von proximal durch den TFCC gestochen, sodass die Kanülenspitze am palmaren Rand des palmaren radioulnaren Bandes sichtbar wird. Der Faden wird mit der Fadenschlinge gefasst und transossär durch die Ulna herausgezogen, c angespannt und zunächst angeklemmt. d Nun wird wiederum in Einwärts-Auswärts-Technik eine zweite Naht zur Reinsertion der dorsalen TFCC-Anteile eingezogen. Hierzu wird eine mit einem 2/OPDS-Faden armierte Kanüle der Größe 2 durch das zentrale Bohrloch in den TFCC eingestochen und der Faden dann mittels einer in eine Kanüle der Größe 1 eingezogenen Fadenschlinge gefasst und dorsal um den PSU herausgeleitet. Beide Nähte werden ohne Distraktionszug, in Neutralrotation des Unterarmes, unter maximaler Spannung auf dem Knochen geknüpft, wodurch der Bandapparat in die vorbereitete Knochenrinne hereingezogen wird. wodurch der Bandapparat in die vorbereitete Knochenrinne hereingezogen wird. Arthroskopisch wird die korrekte Lage der Naht kontrolliert ( $\boldsymbol{\bullet}$ Abb. $\mathbf{6}$ ). Das Retinakulum wird über den Knoten verschlossen. Die Naht wird für 6 Wochen durch eine den Unterarm und den Ellbogen U-förmig umfassende Gipsschiene (Zuckerzangengips) geschützt, die die Rotation und auch die Handgelenksbewegungen einschränkt. Danach wird aktiv remobilisiert, Kraftaufbau und Belastung sollen erst nach 3 Monaten begonnen werden.

Von 2000 bis 2009 wurden 80 Patienten nach dieser Methode operiert. Die ersten 39 Patienten, bis 2007 operiert, wurden von 2 Handchirurgen nachuntersucht, die nicht an der Behandlung beteiligt waren. Die zweite Gruppe von 41 Patienten, von 2007 bis 2009 operiert, wurde aufgrund der von den Operateuren in der Krankenakte eingetragenen Befunde und einer telefonischen Befragung evaluiert.

Behandelt wurden 50 Frauen und 30 Männer mit einem Durchschnittsalter von 30 (14-69) Jahren. Die durchschnittliche Nachbeobachtungszeit der ersten Gruppe betrug 34 (10-83) Monate, die der zweiten Gruppe 29 (12-36) Monate. Nachuntersucht wurden in der ersten Gruppe 66\% und in der zweiten Gruppe $85 \%$ der Patienten. Arthroskopisch zeigten 22 Patienten einen kompletten Abriss des TFCC, 58 einen isolierten Abriss der tiefen Insertion. 90\% der Patienten konnten sich an ein Trauma erinnern. Der Abstand zwischen Trauma und Operation lag zwischen 1 und 180 Monate (durchschnittlich 16 Monate). Klinisch zeigten präoperativ in der ersten Gruppe 20\% der Patienten eine ausgeprägte, die übrigen eine diskrete Instabilität. In der zweiten Gruppe fand sich bei $41 \%$ eine ausgeprägte Instabilität als Ausgangsbefund.

Postoperativ hatten in der ersten Gruppe noch 16\% der nachuntersuchten Patienten eine Restinstabilität, bei den anderen Patienten wurde das DRUG als seitengleich stabil befundet. In der zweiten Gruppe hatten 35\% postoperativ noch eine gewisse Instabilität, die bei jedem dritten (4) Patienten Beschwerden verursachte. Der MMWS besserte sich in beiden Gruppen signifikant: In der ersten Gruppe von präoperativ 60 Punkten auf postoperativ 95 Punkte, in der zweiten Gruppe von 55 auf 90 Punkte. Der DASH-Wert besserte sich in der ersten Gruppe von präope-

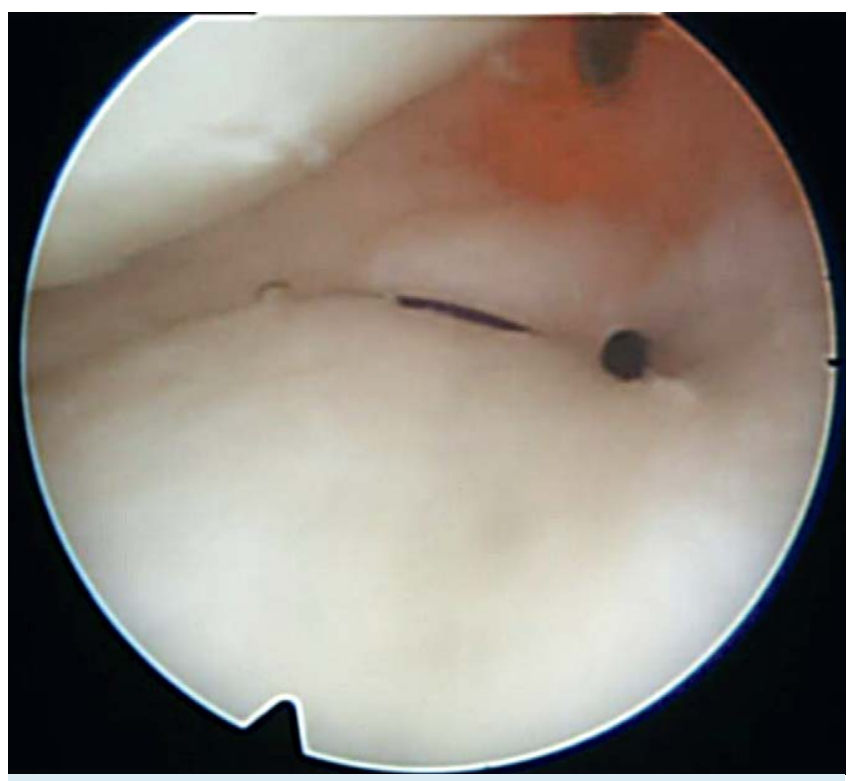

Abb. 6 Rechtes Handgelenk, Arthroskopie von radiokarpal: Der TFCC ist straff refixiert, gleicher Patient wie $\bullet$ Abb. 3. 
rativ 46 Punkte auf postoperativ 5 Punkte, in der zweiten Gruppe von 52 auf 14 Punkte. Der Schmerz, abgeschätzt auf einer NRS von 0 (minimal) bis 10 (maximal), besserte sich in der ersten Gruppe von 6,7 auf 1,1, in der zweiten Gruppe von 6,6 auf 1,4 (jeweils $\mathrm{p}<0,01$ ). Die etwas weniger guten Ergebnisse der zweiten Gruppe sind eventuell darauf zurückzuführen, dass nach den ersten sehr guten Erfahrungen mit dieser Technik die Indikation großzügiger gestellt wurde. Es wurden auch Patienten operiert, bei denen eine ausgeprägte Instabilität bestand. Im Fall der ausgeprägten Instabilität können über den tiefen Abriss in der Fovea hinaus Verletzungen zusätzlicher Strukturen bestehen, insbesondere der ECU-Sehnenscheide. Darauf haben Melone und Nathan schon frühzeitig hingewiesen [2]. Auch Moritomo unterscheidet in mäßige und ausgeprägte Instabilität und stellt die Indikation zur offenen Reinsertion in die Fovea über einen palmaren Zugang nicht im Fall der ausgeprägten Instabilität [5]. Ferner ist bei Ulna-plus-Situation und jeglichem anderem Hinweis auf eine Ulna-Impaktion-Symptomatik, insbesondere Knorpelschäden am proximalen ulnaren Pol des Os lunatum, eine Reinsertion des TFCC ohne gleichzeitige Ulnaverkürzung in der Regel nicht zu empfehlen. Ähnliche Erfahrungen wurden auch von Nakamura mitgeteilt [48]. Auch ein Versuch, geschädigte ulnoradiale Bänder zu reinserieren ist nicht erfolgversprechend. Eine Beurteilung der in der Fovea abgerissenen Bänder über eine Arthroskopie des DRUG ist zweckmäßig. Eine genauere Nachuntersuchung auch unserer später operierten Patienten wird im Moment durchgeführt.

\section{Schlussfolgerung}

$\nabla$

Die ulnaren Läsionen des TFCC lassen sich unterscheiden in rein oberflächliche Abrisse von der Kapsel ohne Instabilität des DRUG, ausgedehntere Abrisse, die zusätzlich die tiefe Insertion der ulnoradialen Bänder in der Fovea betreffen und isolierte Abrisse der in der Fovea ansetzenden Bänder ohne Läsion der oberflächlichen Strukturen. Abrisse in der Fovea gehen mit einer mäßig ausgeprägten Instabilität des DRUG einher. Durch arthroskopisch assistierte Operationen können diese Verletzungen anatomiegerecht rekonstruiert werden. Darüberhinausgehende Verletzungen sind mit einer ausgeprägten Instabilität verbunden und weniger für ein arthroskopisches Vorgehen geeignet. Bei sorgfältiger Indikationsstellung und Beachtung der Kontraindikationen, insbesondere einer knöchernen Inkongruenz des DRUG nach Frakturen und bei Ulna-plus-Situation, sind bei diesen nicht seltenen Verletzungen verlässlich sehr gute und gute Ergebnisse zu erwarten.

\section{Danksagung}

$\nabla$

Für die Untersuchung der bis 2007 operierten Patienten danke ich Frau PD Dr. Nicola Borisch, Baden Baden und Dr. Urs Schmid, Einbeck herzlich.

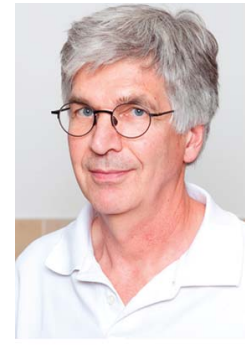

Heinrich-Geert Tünnerhoff, geb. 8.4. 1951, Arzt für Chirurgie - Handchirurgie, Zertifikat Fußchirurgie, Medizinstudium in Würzburg und Bonn von 1970-1976; Weiterbildung Anästhesie in Euskirchen (Dr. Köhnen), Allgemeinchirurgie in Sieglar (Dr. Schneiders) und Gynäkologie in Siegburg ( Prof. Schild) von 1977-1981; allgemeinmedizinische Arbeit und chirurgische Weiterbildung am Huruma Hospital, Rombo ,Tansania, (Dr. Allenspach) 1981-1983; chirurgische Weiterbildung Kreiskrankenhaus Achern (Prof. Zimmermann) 1984-1986; handchirurgische Weiterbildung an der Abteilung für Handchirurgie, plastische und rekonstruktive Chirurgie, DRK Klinik , Baden-Baden (Prof. Haußmann) 1987-1989 und Oberarzt von 1989-2003; 2003 Gründung der Praxis und Belegabteilung für Hand- und Fußchirurgie in Marbach, seit 2006 als Gemeinschaftspraxis mit Dr. Martin Lauffer Arbeitsschwerpunkte:

spezielle Chirurgie und Arthroskopie des Handgelenkes, Rheumachirurgie der Hand,

rekonstruktive Chirurgie des Vorfußes

\section{Interessenkonflikt: Nein}

\section{Literatur}

1 Palmer AK, Werner FW. The triangular fibrocartilage complex of the wrist-anatomy and function. J Hand Surg Am 1981; 6: 153-162

2 Melone CP Jr, Nathan R. Traumatic disruption of the triangular fibrocartilage complex. Pathoanatomy. Clin Orthop Relat Res 1992; 275: 65-73

3 Palmer AK. Triangular fibrocartilage complex lesions: a classification. J Hand Surg Am 1989; 14: 594-606

4 Smith TO, Drew BT, Toms AP et al. The diagnostic accuracy of X-ray arthrography for triangular fibrocartilaginous complex injury: a systematic review and metaanalysis. J Hand Surg Eur 2012; 37: 879-887

5 Moritomo H, Masatomi T, Murase T et al. Open repair of foveal avulsion of the triangular fibrocartilage complex and comparison by types of injury mechanism. J Hand Surg Am 2010; 35: 1955-1963

6 Watanabe A, Souza F, Vezeridis PS et al. Ulnar-sided wrist pain. II. Clinical imaging and treatment. Skeletal Radiol 2010; 39: 837-857

7 Baehser-Griffith P, Bednar JM, Osterman AL et al. Arthroscopic repairs of triangular fibrocartilage complex tears. AORN J 1997; 66: 101-102

8 Thiru RG, Ferlic DC, Clayton ML et al. Arterial anatomy of the triangular fibrocartilage of the wrist and its surgical significance. J Hand Surg Am 1986; 11: 258-263

9 Corso SJ, Savoie FH, Geissler WB et al. Arthroscopic repair of peripheral avulsions of the triangular fibrocartilage complex of the wrist: a multicenter study. Arthroscopy 1997; 13: 78-84

10 Hermansdorfer JD, Kleinman WB. Management of chronic peripheral tears of the triangular fibrocartilage complex. J Hand Surg Am 1991; 16: $340-346$

11 Ruch DS, Anderson SR, Ritter MR. Biomechanical comparison of transosseous and capsular repair of peripheral triangular fibrocartilage tears. Arthroscopy 2003; 19: 391-396

12 Anderson ML, Larson AN, Moran SL et al. Clinical comparison of arthroscopic versus open repair of triangular fibrocartilage complex tears. J Hand Surg 2008; 33: 675-682

13 de Araujo W, Poehling GG, Kuzma GR. New Tuohy needle technique for triangular fibrocartilage complex repair: preliminary studies. Arthroscopy 1996; 12: 699-703

14 Millants P, De Smet L, Van Ransbeeck H. Outcome study of arthroscopic suturing of ulnar avulsions of the triangular fibrocartilage complex of the wrist. Chir Main 2002; 21: 298-300

15 Haugstvedt JR, Husby T. Results of repair of peripheral tears in the triangular fibrocartilage complex using an arthroscopic suture technique. Scand J Plast Reconstr Surg Hand Surg 1999; 33: 439-447

16 Trumble TE, Gilbert M, Vedder N. Isolated tears of the triangular fibrocartilage: management by early arthroscopic repair. J Hand Surg Am 1997; 22: 57-65 
17 Estrella EP, Hung LK, Ho PC et al. Arthroscopic repair of triangular fibrocartilage complex tears. Arthroscopy 2007; 23: 729-737, 737 e721

18 Tünnerhoff HG, Haussmann P. Wann ist die arthroskopische Refixation des Discus ulnocarpalis bei ulnarem Abriss indiziert. Handchir Mikrochir Plastische Chir 2001; 33: 239-244

19 Wedemeyer A, Wedemeyer C, Heckelei $W$ et al. Arthroskopische Refixation von Traumatischen1B_Läsionen des Discus ulnocarpalis - eine retrospektive Studie. Handchir Mikrochir Plastische Chir 2009; 41: 135-140

20 Gofton $W T$, Gordon $K D$, Dunning $C E$ et al. Soft-tissue stabilizers of the distal radioulnar joint: an in vitro kinematic study. J Hand Surg Am 2004; 29: 423-431

21 Kakar S, Carlsen BT, Moran SL et al. The management of chronic distal radioulnar instability. Hand clinics 2010; 26: 517-528

22 Stuart PR, Berger RA, Linscheid RL et al. The dorsopalmar stability of the distal radioulnar joint. J Hand Surg Am 2000; 25: 689-699

23 Kihara $H$, Short WH, Werner FW et al. The stabilizing mechanism of the distal radioulnar joint during pronation and supination. J Hand Surg Am 1995; 20: 930-936

24 Hagert CG. Distal radius fracture and the distal radioulnar joint - anatomical considerations. Handchir Mikrochir Plastische Chir 1994; 26 : $22-26$

25 Nakamura T, Takayama S, Horiuchi $Y$ et al. Origins and insertions of the triangular fibrocartilage complex: a histological study. J Hand Surg 2001; 26B: 446-454

26 Kleinman WB. Stability of the distal radioulna joint: biomechanics, pathophysiology, physical diagnosis, and restoration of function what we have learned in 25 years. J Hand Surg Am 2007; 32: 1086-1106

27 Atzei A. New trends in arthroscopic management of type 1-B TFCC injuries with DRUJ instability. J Hand Surg 2009; 34B: 581-591

28 Tay SC, Berger RA, Parker WL. Longitudinal split tears of the ulnotriquetral ligament. Hand Clin 2010; 26: 495-501

29 Moritomo HM, Tsuyoshi M, Sayuri A et al. Change in the length of the ulnocarpal ligaments during radiocarpal motion: Possible impact on triangular fibrocartilage complex foveal tears. J Hand Surg Am 2008; 33: $1278-1286$

30 Moritomo HM, Murase T, Arimitsu S et al. Change in the Length of the ulnocarpal ligament during radiocarpal motion: Possible impact on triangular fibrocartilage complex foveal tears. J Hand Surg Am 2010; 35: 1955-1963

31 Nakamura T. Radial side tears of the triangular fibrocartilage complex. In: Pinal P. Arthroscopic Management of Distal Radius Fractures. Heidelberg: Springer: 2010; 89-98

32 Sagerman $S D$, Short $W$. Arthroscopic repair of radial-sided triangular fibrocartilage complex tears. Arthroscopy 1996; 12: 339-342

33 Iida A, Omokawa S, Moritomo H et al. Effect of wrist position on distal radioulnar joint stability: A biomechanical study. J Orthop Res 2014; 32: 1247-1251

34 Tay SC, Tomita K, Berger RA. The "ulnar fovea sign" for defining ulnar wrist pain: an analysis of sensitivity and specificity. J Hand Surg Am 2007; 32: 438-444

35 Reagan DS, Linscheid RL, Dobyns JH. Lunotriquetral sprains. J Hand Surg Am 1984; 9: 502-514

36 Kip PC, Peimer CA. Release of the sixth dorsal compartment. J Hand Surg Am 1994; 19: 599-601
37 Burkhart SS, Wood MB, Linscheid RL. Posttraumatic recurrent subluxation of the extensor carpi ulnaris tendon. J Hand Surg Am 1982; 7: 1-3

38 Ruland RT, Hogan CJ. The ECU synergy test: an aid to diagnose ECU tendonitis. J Hand Surg Am 2008; 33: 1777-1782

39 Adams BD. Effects of radial deformity on distal radioulnar joint mechanics. J Hand Surg Am 1993; 18: 492-498

40 Pogue D, Viegas SF, Patterson RM et al. Effects of distal radius fracture malunion on wrist joint mechanics. J Hand Surg Am 1990; 15: 721-727

41 Dy CJ, Jang E, Taylor SA et al. The Impact of Coronal Alignment on Distal Radioulnar Joint Stability Following Distal Radius Fracture. J Hand Surg Am 2014; 39: 1264-1272

42 Hahn P, Hausler A, Bruckner T et al. Wertigkeit der MRT hinsichtlich TFCC-Läsionen außerhalb klinischer Studien. Handchir Mikrochir Plast Chir 2012; 44: 310-313

43 Amrami KK, Moran SL, Berger RA et al. Imaging the distal radioulnar joint. Hand Clin 2010; 26: 467-475

44 MacLennan AJ, Nemechek NM, Waitayawinyu T et al. Diagnosis and anatomic reconstruction of extensor carpi ulnaris subluxation. J Hand Surg Am 2008; 33: 59-64

45 Park MJ, Jagadish A, Yao J. The rate of triangular fibrocartilage injuries requiring surgical intervention. Orthopedics 2010; 33: 806-813

46 Sarmiento A, Cooper JS, Sinclair WF. Forearm fractures. Early functional bracing - A preliminary report. J Bone Joint Surg Am 1975; 57: 297-304

47 Millard GM, Budoff JE, Paravic V et al. Functional bracing for distal radioulnar joint instability. J Hand Surg Am 2002; 27: 972-977

48 Nakamura T, Sato K, Okazaki $M$ et al. Repair of foveal detachment of the triangular fibrocartilage complex: open and arthroscopic transosseous techniques. Hand Clin 2011; 27: 281-290

49 Slutsky DJ. Arthroscopic evaluation of the foveal attachment of the triangular fibrocartilage. Hand Clin 2011; 27: 255-261

50 Shinohara T, Tatebe M, Okui N et al. Arthroscopically assisted repair of triangular fibrocartilage complex foveal tears. J Hand Surg Am 2013; 38: 271-277

51 Iwasaki N, Nishida K, Motomiya $M$ et al. Arthroscopic-assisted repair of avulsed triangular fibrocartilage complex to the fovea of the ulnar head: a 2- to 4-year follow-up study. Arthroscopy 2011; 27: 1371-1378

52 Garcia-Elias $M$, Hagert E. Surgical approaches to the distal radioulnar joint. Hand Clin 2010; 26: 477-483

53 Wysocki RW, Richard MJ, Crowe M et al. Arthroscopic treatment of peripheral triangular fibrocartilage complex tears with the deep fibers intact. J Hand Surg Am 2012; 37: 509-516

54 Wolf $M B$, Haas A, Dragu A et al. Arthroscopic repair of ulnar-sided triangular fibrocartilage complex (Palmer Type 1B) tears: a comparison between short- and midterm results. J Hand Surg Am 2012; 37: 2325-2330

55 Cardenas-Montemayor E, Hartl JF, Wolf MB et al. Subjective and objective results of arthroscopic debridement of ulnar-sided TFCC (Palmer type 1B) lesions with stable distal radio-ulnar joint. Arch Orthop Trauma Surg 2013; 133: 287-293

56 Nishizuka T, Tatebe $M$, Hirata $H$ et al. Simple debridement has little useful value on the clinical course of recalcitrant ulnar wrist pain. Bone Joint J 2013; 95-B: 1687-1696 